\title{
A Negotiation Pricing Model for Innovation Services Based on the Multiobjective Genetic Algorithm
}

\author{
Yan Zhou ${ }^{1}$, Yue Li ${ }^{1}$, Yunxing Zhang,** \\ 1 Harbin University of Science and Technology, Harbin, 150080, China; \\ 2 Harbin Engineering University, Harbin, 150001, China; \\ * Correspondence: zhangyunxing@hrbeu.edu.cn
}

\begin{abstract}
Service pricing is a bottleneck in the development of innovation services, as it is the issue of most concern between the suppliers and demanders. In this paper, a negotiation pricing model that is based on the multiobjective genetic algorithm is developed for innovation service pricing. Regarding the service pricing process as a multiobjective problem, the objective functions, which include the service price, service efficiency, and service quality, for suppliers and demanders are constructed. As the solution of a multiobjective problem is typically a series of alternatives, another negotiation process is necessary for determining the final decision. A learning strategy is adopted during the negotiation process to simulate reality. Finally, the model is implemented for an innovation service transaction, the objective of which is to identify the optimal price plan. The results demonstrate that the model can provide quantitative decision support for the pricing of an innovation service and ultimately yield a win-win result for both the supplier and demander of the innovation service. Furthermore, the influence of the parameters during the negotiation process is analyzed in detail. The effects of the learning strategy on accelerating the negotiation process, as well as the chosen of reasonable parameters are given.
\end{abstract}

Keywords: innovation service; pricing model; multiobjective problem; genetic algorithm; negotiating strategies

\section{Introduction}

A 'three-step' strategy is proposed by the Chinese government in the 'Outline of the National Strategy of Innovation-Driven Development' [1], via which by 2020, China will become an innovation-oriented country; by 2030, it will be among the top innovation-oriented countries; and by 2050, it will be a world power in science and technology innovation. To realize the objective, industries with strong innovation vitality were incorporated into strategic emerging industries in China [2], which is the key to realizing the strategic deployment of industrial innovation driving [3].

Under the strategy of innovation-driven development, innovation service emerges. Innovation service is the provision of single or combined services, such as research and development service, intellectual property service, and basic technology service for industrial innovation demand, especially for strategic emerging industries. The subjects of innovation service include science and technology intermediaries [4, 5], innovation service platforms [6], science and technology resource sharing platforms [7], and knowledge service intermediaries [8], among others. Resource advantages and service functions are combined among these subjects to provide service support for industrial innovation and to increase industrial innovation efficiency effectively.

The precondition for an innovation service to realize its value is adoption by innovation enterprises [9]. Typically, the price of an innovation service is the main factor that determines its successful adoption by innovation enterprises. Therefore, the pricing of innovation services could be the key to the transaction of innovation services, which, in turn, will affect the overall development of innovation services. Therefore, the pricing of innovation services reasonably and the maximization of the benefits of both the suppliers and demanders of innovation services have become urgent problems to be solved. 
In earlier studies, the pricing of services mostly refers to the pricing method of commodities, and the prices are determined based on the supply-demand relationship [10], and the service cost. Kung et al. (2017) attempted to determine service prices using game theory [11]. Li et al. (2016) provided a pricing framework for big data services that was based on the comprehensive service quality, service time, and matching degree of supply and demand [12]. With the development of blockchain technology, researchers began to use it to mediate the pricing of services [13]. However, the service pricing methods in the above studies are all set by the suppliers. Due to the frequent interaction between the suppliers and demanders in innovation services, the demander also plays an important role in the price-setting process; hence, the simple method is difficult to adapt to the particularity of innovation services.

In this study, the pricing process of innovation services is regarded as the process of value co-creation for both the supplier and the demander [14]. The pricie of an innovation service is reasonable if the supplier and demander realize a win-win scenario. Therefore, the utilities of the supplier and demander can be regarded as objective functions, and the pricing of innovation services is essentially a multiobjective decision-making problem. However, the solution of a multiobjective problem is usually a series of alternatives; thus, another process is necessary for the final decision.

In this paper, the Non-Dominated Sorting Genetic Algorithm-II (NSGA-II) algorithm is utilized to solve the multiobjective problem to identify the Pareto boundary (the previously discussed 'series of alternatives'). To make the final decision, a negotiation process is performed within the Pareto boundary. A learning strategy is adopted during the negotiation process to simulate reality. The remainder of this paper is structured as follows: The pricing process of innovation services is analyzed theoretically in Section 2, and a pricing model for innovation services is exhibited. Section 3 presents the algorithm of the model in detail. In Section 4, an application of the model is described, and the influence of the parameters during the negotiation process is discussed in Section 5. Finally, the conclusions are provided in Section 6.

\section{Theoretical Analysis \& Modeling}

In this section, the problem of innovation service pricing is analyzed theoretically. Three hypotheses are formulated, based on which the model is constructed.

A practical service pricing process typically involves more than one demander and supplier. However, the multiple demanders and suppliers can be regarded as the superposition of single demander and supplier pricing problems. Therefore, Hypothesis 1 is proposed:

Hypothesis 1: The numbers of demanders and suppliers of the service are both 1 and are denoted as $\mathrm{D}$ and $\mathrm{S}$, respectively.

In a practical service pricing process, the quality and efficiency of the service will also affect the price acceptance of the demander and supplier, in addition to the price of services ${ }^{[11,12]}$. Usually, both the supplier and demander have acceptance ranges for each attribute. Therefore, Hypothesis 2 is proposed:

Hypothesis 2: The set of service attributes includes the service price, service efficiency, and service quality and is denoted as $\Phi=\left\{\Phi_{1}, \Phi_{2}, \Phi_{3}\right\}$. The value of $\Phi_{j}$ is denoted by $\phi_{j}(j \in(1,2,3))$. The acceptable interval for the demander $\mathrm{D}$ on $\Phi_{j}$ is denoted by $\left[\phi_{D j}{ }^{{ }^{\min }},{ }_{D_{D j}}{ }^{\max }\right]$, and the acceptable interval for the supplier $S$ on $\Phi_{j}$ is denoted by $\left[\phi_{s_{j}}^{\text {min }}, \phi_{s_{j}}^{{ }^{\max }}\right]$.

The levels of importance for each attribute of the service differ between the demander and supplier. The weight of each attribute must be considered. Therefore, Hypothesis 3 is proposed:

Hypothesis 3: The demander and supplier specify the set of weights for each attribute according to their scenarios, which are recorded as $\Omega^{D}=\left(\begin{array}{lll}\omega_{1}^{D}, & \omega_{2}^{D}, & \omega_{3}^{D}\end{array}\right)$ and $\Omega^{S}=\left(\begin{array}{lll}\omega_{1}^{S}, & \omega_{2}^{S}, & \omega_{3}^{S}\end{array}\right)$ and satisfy:

$$
\sum_{j=1}^{3} \omega_{j}^{D}=\sum_{j=1}^{3} \omega_{j}^{S}=1
$$

Under the above hypotheses, a model for analyzing innovative service pricing can be developed. The model is composed of several steps (Figure 1), which are described as follows: 
Step 1: Set the initial parameters of the supplier and demander, which include the acceptable intervals $\left[\phi_{D_{j}}{ }^{\min }, \phi_{D_{j}}{ }^{\max }\right]$ and $\left[\phi_{S_{j}}{ }^{\min }, \phi_{S_{j}}^{\max }\right](j \in(1,2,3))$ and the corresponding sets of weights $\Omega^{D}$ and $\Omega^{s}$.

Step 2: Construct the objective functions of the supplier and demander, which are denoted as $F_{D}(\Phi)$ and $F_{S}(\Phi)$.

Step 3: Find the solution of the multiobjective optimization problems (Pareto boundary).

Step 4: Obtain the final decision $\phi_{j}(j \in(1,2,3))$ via the chosen negotiation strategy on the Pareto boundary.

Set initial parameters

Construct objective functions

Get

Pareto boundary

Choose negotiation strategy

Obtain

final decision

Figure 1. Flowchart of the innovation service pricing model.

\section{Algorithm Design}

\subsection{Objective function construction}

The benefit is usually the objective of both the supplier and demander. In the model, the total benefit is assumed to be the sum of the benefits that are obtained from the service price, service efficiency, and service quality. Furthermore, it is assumed that there is no overlap between the benefits of the three parts, namely, that the benefit can be linearly superimposed. Based on the comprehensive benefit calculation formula that was proposed by Raiffa [15], the benefit functions for the supplier and demander are:

$$
\begin{gathered}
F_{D}(\Phi)=\sum_{j=1}^{3} \omega_{j}^{D} \nu_{j}^{D}\left(\phi_{j}\right) \\
F_{S}(\Phi)=\sum_{j=1}^{3} \omega_{j}^{S} v_{j}^{S}\left(\phi_{j}\right)
\end{gathered}
$$

where $v_{j}^{D}$ and $v_{j}^{S}$ are the contributions of the attribute $j$ to the demander and supplier, respectively. The calculation method for $v_{j}^{D}$ and $v_{j}^{s}$ is defined as follows:

$$
v_{j}= \begin{cases}\frac{\phi_{j}^{\max }-\phi_{j}}{\phi_{j}^{\max }-\phi_{j}^{\min }} & \text { For 'Cost' } \\ \frac{\phi_{j}-\phi_{j}^{\min }}{\phi_{j}^{\max }-\phi_{j}^{\min }} & \text { For 'Benefit' }\end{cases}
$$

Here, 'cost' and 'benefit' are tags that are attached to the attributes. The tag of an attribute indicates whether the increase of the attribute will lead to an increase of the cost or benefit. Each attribute could have different tags for the demander and supplier, as presented in Table 1. 
Table 1. Tags of each attribute for the demander and supplier.

\begin{tabular}{ccc}
\hline & Demander & Supplier \\
\hline Service price & Cost & Benefit \\
Service efficiency & Benefit & Cost \\
Service quality & Benefit & Cost \\
\hline
\end{tabular}

125

126

127

128

129

130

131

To maximize the benefits of both the supplier and demander and regarding the acceptable intervals of the attributes, which include the service price, service efficiency, and service quality, as constraints, the following multiobjective problem can be formulated:

$$
\max F(\Phi)=\left[\sum_{j=1}^{3} \omega_{j}^{D} v_{j}^{D}\left(\phi_{j}\right), \sum_{j=1}^{3} \omega_{j}^{S} v_{j}^{S}\left(\phi_{j}\right)\right]
$$

Subject to:

$$
\begin{aligned}
& \phi_{D j}{ }^{\min } \leq \phi_{j} \leq \phi_{D_{j}}^{\max } \quad j=1,2,3 \\
& \phi_{S_{j}}{ }^{\min } \leq \phi_{j} \leq \phi_{S_{j}}{ }^{\max } \quad j=1,2,3
\end{aligned}
$$

\subsection{Identification of the Pareto boundary}

Equations (4-5) define a multiobjective optimization problem, which can usually be solved via the genetic algorithm [16,17], ant colony algorithm [18], particle swarm optimization algorithm [19] or simulated annealing algorithm [20]. In this paper, one of the most widely used multiobjective optimization methods, namely, non-dominated sorting genetic algorithm II (NSGA-II), is used to solve the noninferior solution set of the problem.

NSGA-II is a multiobjective optimization method that is based on the genetic algorithm. The basic process of NSGA-II is illustrated in Figure 2, and detailed information about the algorithm is provided in references [21] and [22].

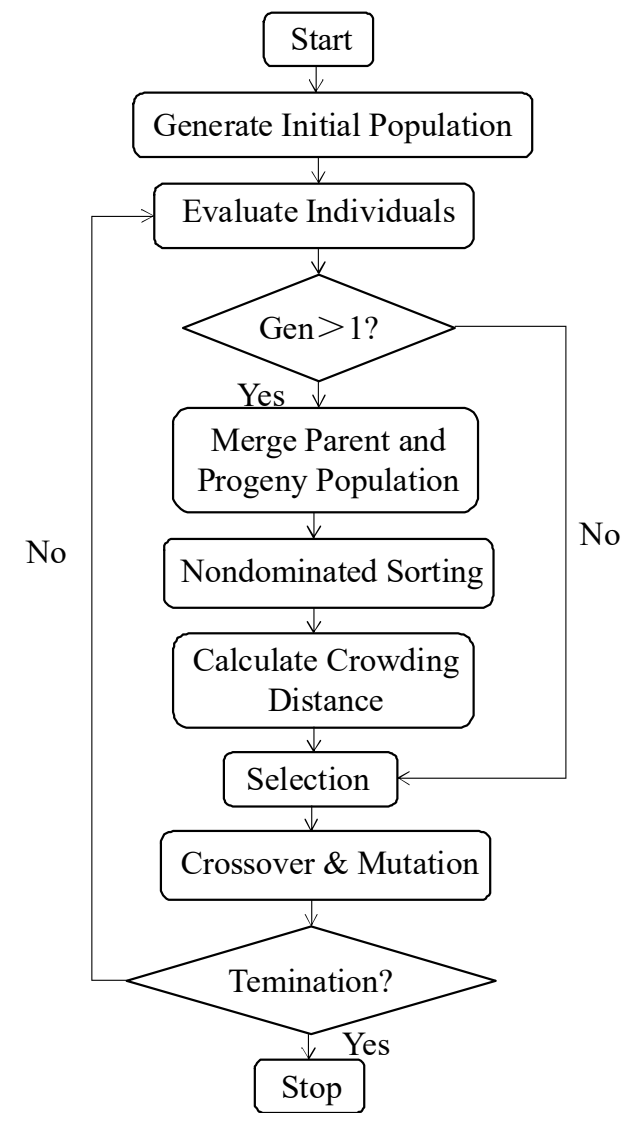

142 Figure 2. Flowchart of NSGA-II algorithm. 
Via the NSGA-II algorithm, the Pareto boundary of a multiobjective problem is obtained. The Pareto boundary is composed of a series of points, and each point represents an attribute combination $O=\left\{\begin{array}{lll}O_{1}, O_{2}, & O_{3}\end{array}\right\}$ and corresponding expected utilities for the supplier and demander $U=\left\{U_{S}, U_{D}\right\}$.

\subsection{Negotiation on the Pareto boundary}

A series of alternatives (Pareto boundary) are obtained via NSGA-II in Section 3.2. In this section, a negotiation is conducted to make the final decision. The classic negotiation strategy function that was proposed by Faratin [23] is adopted, in combination with the learning strategy, to complete the negotiation process.

In Faratin's method, the supplier and demander reached an agreement via anticipation and gradual concessions. For each negotiation, the expected utility $E U(t)$ is:

$$
E U(t)=\max -\alpha(t)(\max -\min )
$$

Here, $t$ is the number of negotiations; max and min are the maximum and minimum values, respectively, of the acceptable utilities for the supplier or demander of innovation services; and $\alpha(t)$ is the concession coefficient, which has the following form:

$$
\alpha(t)=e^{\left[1-\frac{\min \left(t, t_{\max }\right)}{t_{\max }}\right]^{\beta} \ln \kappa}
$$

in which $t_{\max }$ is the maximum number of negotiations; $\kappa$ is the initial concession coefficient, which corresponds to the initial value of $\alpha(t)$; and $\beta$ is the concession speed control parameter, according to which the degree of aggressiveness of the negotiation strategy that is adopted by both parties is determined. The value of $\alpha(t)$ as a function of the number of negotiations $t$ is plotted in Figure 3 for various $\beta$ values.

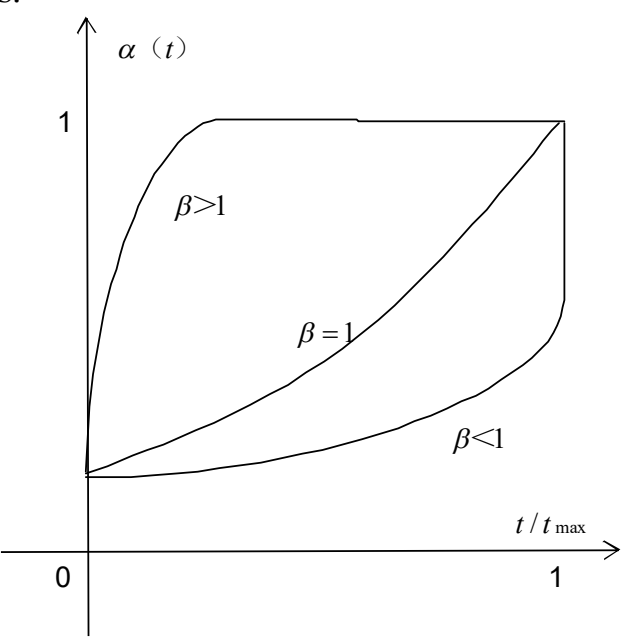

Figure 3. $\alpha(t)$ as a function of the number of negotiations for various $\beta$ values $(\kappa=0.2)$.

The learning strategy attempts to adjust the value of $\beta$ automatically according to the concession of the other party [24]. The method was demonstrated to accelerate and increase the robustness of the negotiation process. Considering the supplier as an example, $U_{D \rightarrow s}^{t}-U_{D \rightarrow S}^{t-1}$ is the difference between the utilities of the last two proposals; here, $U_{D \rightarrow S}^{t}$ represents the supplier's utility with the demander's bids. Define the concession rate $\theta$ as the ratio of the differences:

$$
\theta=\left(U_{D \rightarrow S}^{t}-U_{D \rightarrow S}^{t-1}\right) /\left(U_{D \rightarrow S}^{t-1}-U_{D \rightarrow S}^{t-2}\right)
$$

Now, we discuss the automatic adjustment of the value of $\beta$. We return to the definition of $\theta$ (equation 8). If $\theta>1$, the concession margin of the demander is gradually increasing. Then, the supplier should suitably reduce its concession margin to avoid unnecessary concession and to increase its final utility. If $\theta=1$, the demander is steadily acquiescing. Thus, the supplier should hold the $\beta$ value constant and continue to wait and see. If $\theta<1$, the demander is reducing its concession. Now, the supplier should make a large concession to approach the demander's expected 
bid to increase the success rate of the negotiation. After a series of tests, the value of $\beta=1 / \theta$ is adopted due to its simplicity and effectiveness.

The negotiation is conducted on the Pareto boundary that was identified via the NSGA-II multiobjective optimization algorithm. The supplier and demander compare their actual and expected utilities on the Pareto boundary and negotiate until an agreement is reached. The process is described as follows (and a flowchart is presented in Figure 4):

Step 1: Based on the Pareto boundary, the supplier bids on its highest utility. The utility of the supplier is defined as $U_{S}^{t}$, and the corresponding combination of bidding attributes is $O^{t}$. Proceed to step 2.

Step 2: After receiving a bid from the supplier, the demander obtains its own utility $U_{D}^{t}$ with the Pareto boundary. Then, the demander compares $U_{D}^{t}$ with $E U_{D}^{t}$, which can be obtained from the concession function (equation (6)). If $E U_{D}^{t}$ exceeds $U_{D}^{t}$, then proceed to step 3; otherwise (if the current utility exceeds the expected utility), proceed directly to step 6.

Step 3: The demander rejects the bid of the supplier as it does not meet the demander's expectation. Then, on the basis of $E U_{D}^{t}$, the demander finds the utility $U_{D}^{t+1}$ that is closest to its $E U_{D}^{t}$ on the Pareto boundary and provides the corresponding $O^{t+1}$ as the bid. Proceed to step 4.

Step 4: The supplier receives the new bid and obtain the corresponding utility $U_{S}^{t+1}$. Similar to step 2, the supplier compares $U_{S}^{t+1}$ with $E U_{S}^{t+1}$, which can be obtained from the concession function (equation (6)). If $E U_{S}^{t+1}$ exceeds $U_{S}^{t+1}$, then proceed to step 5; otherwise (if the current utility exceeds the expected utility), proceed directly to step 6.

Step 5: The supplier rejects the bid of the demander as it does not meet the supplier's expectation. Then, on the basis of $E U_{S}^{t+1}$, the supplier finds the utility $U_{S}^{t+2}$ that is closest to its $E U_{S}^{t+1}$ on the Pareto boundary and provides the corresponding $O^{t+2}$ as the bid. Return to step 2.

Step 6: The bid now meets the supplier's and demander's expectations. An agreement is reached, and the negotiation is terminated.

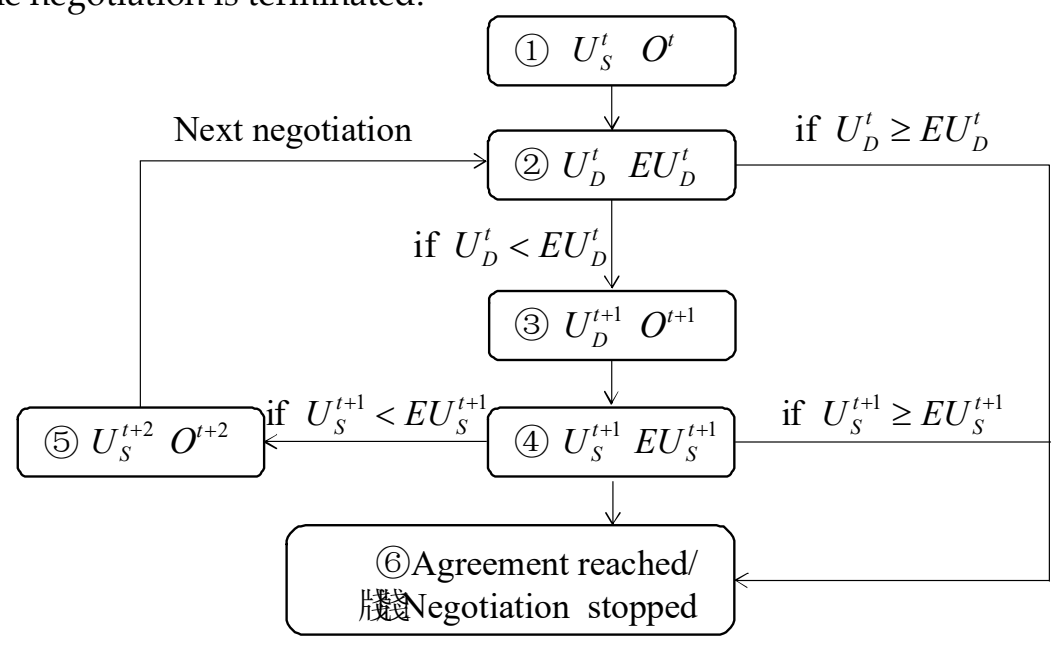

Figure 4. Flowchart of the negotiation process.

\section{Model Implementation}

In this section, the proposed model is implemented in a service pricing problem. To evaluate the price of an $\mathrm{R} \& \mathrm{D}$ service, an innovation service platform selects one representative company from potential suppliers and demanders, which are denoted as $S$ and $D$, respectively. The platform collects the weights and acceptable intervals for the service price, service efficiency and service quality of the R \& D service for S and D. Relevant parameters for supplier and demander in the R \& $\mathrm{D}$ service pricing are presented in Table 2.

Table 2. Parameters for the supplier and demander in the R \& D service pricing.

Attributes $\quad \mathrm{R} \& \mathrm{D}$ service demander $\mathrm{D}$

R \& D service supplier $S$ 
213

214

215

216

\begin{tabular}{ccccc}
\hline & Weights & $\begin{array}{c}\text { Acceptable } \\
\text { intervals }\end{array}$ & Weights & $\begin{array}{c}\text { Acceptable } \\
\text { intervals }\end{array}$ \\
Service price & 0.5 & {$[6,10]$} & 0.7 & {$[8,10]$} \\
Service efficiency & 0.2 & {$[2,6]$} & 0.1 & {$[1,5]$} \\
Service quality & 0.3 & {$[4,6]$} & 0.2 & {$[3,6]$} \\
\hline
\end{tabular}

According to formula (3), the utilities of each attribute for the supplier and demander of the R \& D service are calculated:

$$
\begin{aligned}
& v_{1}^{D}=\frac{\phi_{D 1}^{\max }-\phi_{1}}{{\phi_{D 1}{ }^{\max }-\phi_{D 1}{ }^{\min }}^{2}}=\frac{10-\phi_{1}}{10-6}=\frac{10-\phi_{1}}{4} \\
& v_{2}^{D}=\frac{\phi_{2}-\phi_{D 2}^{\min }}{\phi_{D 2}^{\max }-\phi_{D 2}^{\min }}=\frac{\phi_{2}-2}{6-2}=\frac{\phi_{2}-2}{4} \\
& v_{3}^{D}=\frac{\phi_{3}-\phi_{D 3}^{\min }}{\phi_{D 3}^{\max }-\phi_{D 3}^{\min }}=\frac{\phi_{3}-4}{6-4}=\frac{\phi_{3}-4}{2} \\
& v_{1}^{S}=\frac{\phi_{1}-\phi_{S 1}^{\min }}{\phi_{S_{1}}^{\max }-\phi_{S_{1}}^{\min }}=\frac{\phi_{1}-8}{10-8}=\frac{\phi_{1}-8}{2} \\
& v_{2}^{S}=\frac{\phi_{S 2}^{\max }-\phi_{2}}{\phi_{S_{2}}^{\max }-\phi_{S_{2}}^{\min }}=\frac{5-\phi_{2}}{5-1}=\frac{5-\phi_{2}}{4} \\
& v_{3}^{S}=\frac{\phi_{S 3}^{\max }-\phi_{3}}{\phi_{S 3}^{\max }-\phi_{S 3}^{\min }}=\frac{6-\phi_{3}}{6-3}=\frac{6-\phi_{3}}{3}
\end{aligned}
$$

According to formulas (4) and (5), the following objective functions and constraints can be obtained:

$$
\begin{aligned}
& \max F_{D}(\Phi)=0.5 \cdot \frac{10-\phi_{1}}{4}+0.2 \cdot \frac{\phi_{2}-2}{4}+0.3 \cdot \frac{\phi_{3}-4}{2} \\
& \max F_{S}(\Phi)=0.7 \cdot \frac{\phi_{1}-8}{2}+0.1 \cdot \frac{5-\phi_{2}}{4}+0.2 \cdot \frac{6-\phi_{3}}{3}
\end{aligned}
$$

subject to:

The NSGA-II algorithm is utilized to solve the above multiobjective optimization problem. The relevant parameter settings of the NSGA-II algorithm are presented in Table 3. The Pareto boundary is identified, as shown in Figure 5.

Table 3. Relevant parameters of the NSGA-II algorithm.

\begin{tabular}{cc}
\hline Pareto Fraction & 0.6 \\
\hline Population Size & 1000 \\
Generations & 2000 \\
Stall GenLimit & 2000 \\
TolFun & $1 \mathrm{e}-100$ \\
\hline
\end{tabular}




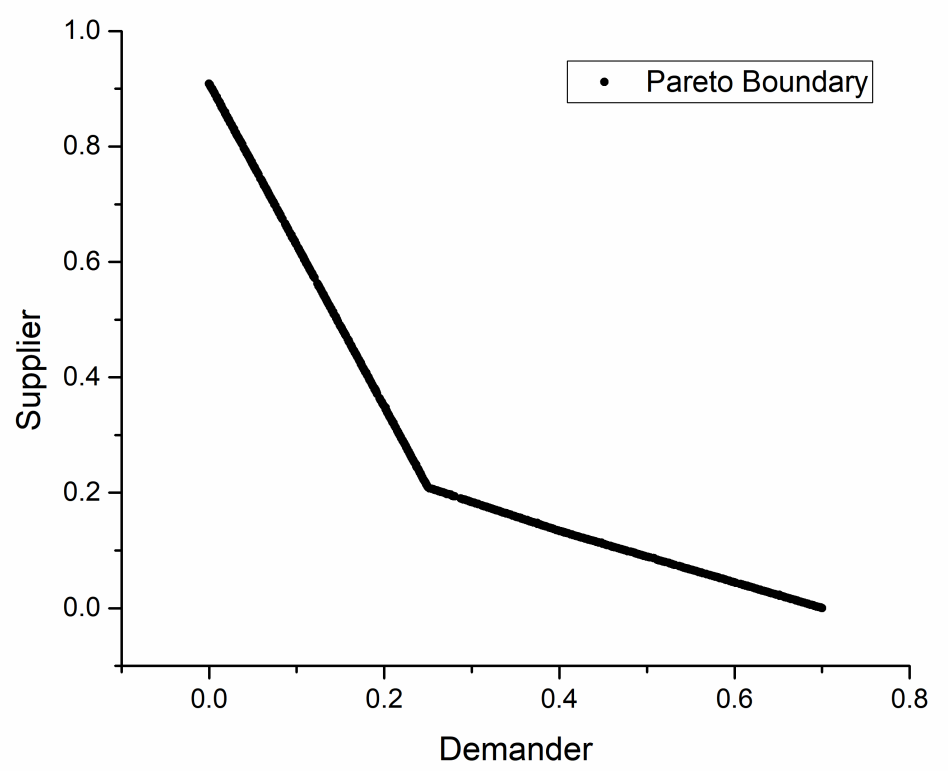

Figure 5. Pareto boundary for the problem.

Based on the Pareto boundary, the service supplier and demander negotiate service prices, service quality, and service efficiency. The actual and expected utilities of the supplier and demander change with the number of negotiations, as shown in Figure 6.

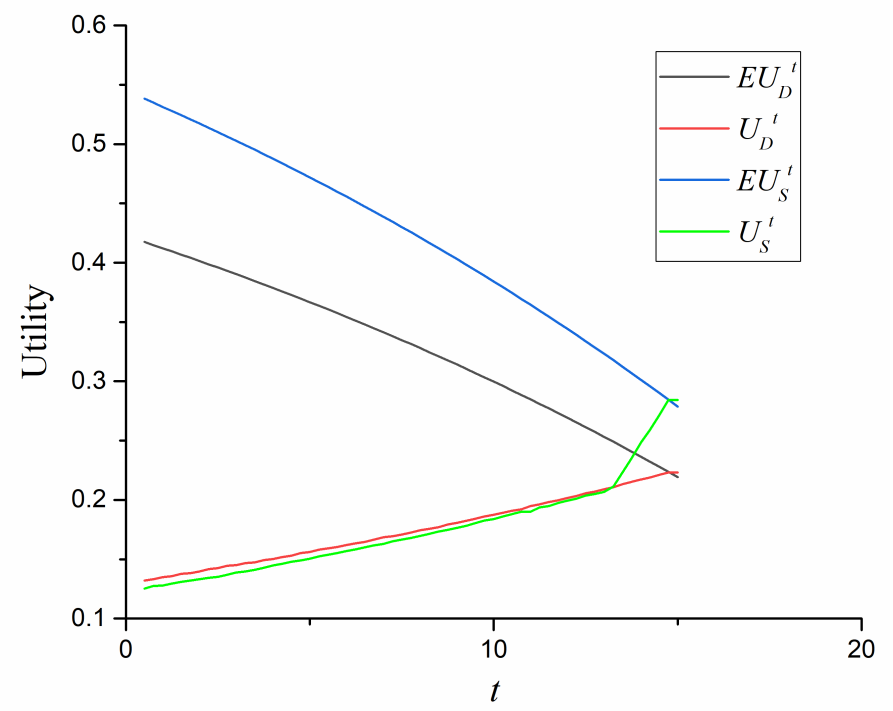

Figure 6. Actual and expected utilities of the supplier and demander as functions of the number of negotiations

As shown in the figure, the actual utilities for the supplier and demander reached the expected utility at the 15th negotiation, namely, an agreement was reached. The final bid for the 15th negotiation was 8.4228; thus, the price of the R \& D service was 8.4228.

5. Discussion on Negotiation Process

The learning strategy and some user-defined parameters are adopted during the negotiation process, while the influence of them has not been fully discussed. The objective of this section is to discuss the influence of learning strategy and user-defined parameters in detail. The results of the problem in section 4 is considered as the basis, which means that the Pareto boundary is kept same as above for all the following discussions. 


\subsection{Learning strategy}

First, we study on the influence of learning strategy. For simplicity, the control parameters including (initial concession parameter $\kappa$ and concession speed control parameter $\beta$ ) are the same as section 4 . The learning strategy is simply shut down or not. The results of the negotiation process is shown in figure 7. The number of negotiations for with and without learning strategy are 15 and 60 , respectively. The final results are the same for the two conditions. Thus, the negotiation process is greatly accelerated with the learning strategy with no change on the final results.

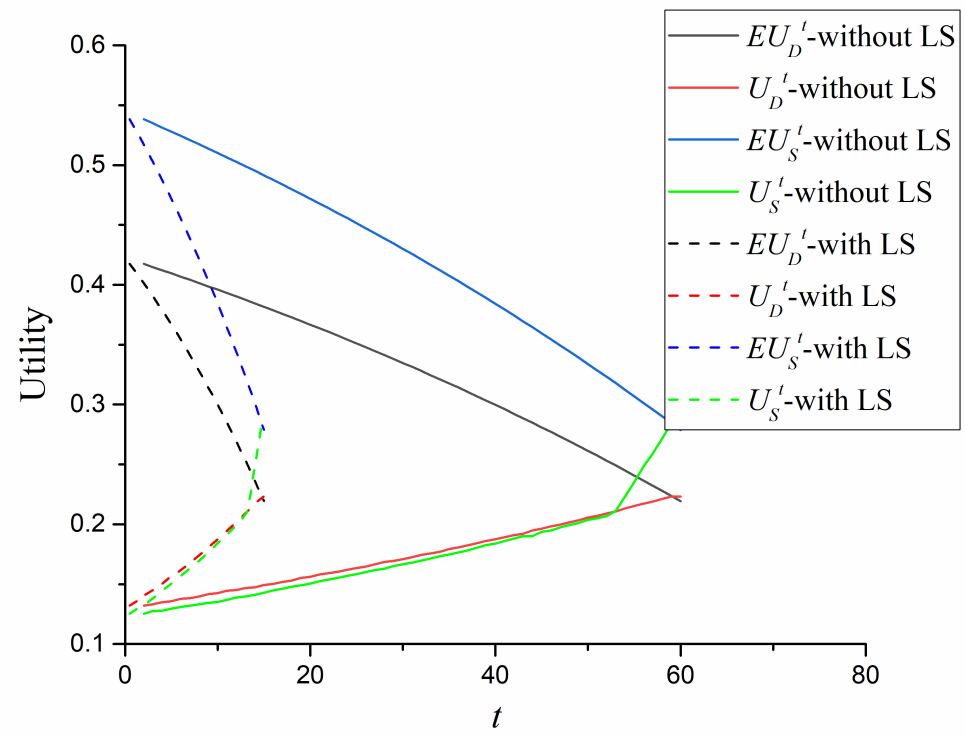

Figure 7. Utilities of the supplier and demander as functions of of the number of negotiations with or without learning strategy

\subsection{Initial concession parameter $\kappa$ and concession speed control parameter $\beta$}

The initial concession parameter $\kappa$ and concession speed control parameter $\beta$ are two important parameters during the negotiation process. To analyze them in detail, $\kappa$ ranging from 0.1 to 1.0 and $\beta$ ranging from 0.2 to 1.8 are selected. The interval of value range for $\kappa$ and $\beta$ are 0.1 and 0.2 , respectively. The $t_{\max }$ in equation (6) is set 100 for all the simulations, which we think is reasonable for reality.

The results of iteration number and the utilities of the demander and supplier for various combinations of $(\kappa, \beta)$ are given in table 4. It is not hard to find that a larger initial concession parameter $\kappa$ and concession speed control parameter $\beta$ will lead to a smaller iteration number. It is noted that when the value of $\kappa$ is larger than 0.6, program crashed. However, the accuracy of the results need to be checked. Here the results with very large $t_{\max }$ (in fact we use $t_{\max }=10000$ ) and very small $\kappa$ and $\beta(\kappa=0.05$ and $\beta=0.05)$ as the reference results. The utilities of the demander and supplier are 0.221799 and 0.288188 , respectively. The following equation is is adopted to compute the relative error

$$
\text { Relative Error }=\frac{\left|D e .-D e_{\text {ref }}\right|}{D e_{\text {ref }}}+\frac{\left|S u .-S u_{\text {ref }}\right|}{S u_{\text {ref }}}
$$

Here De. and Se. Indicates the utilities of the demander and supplier, respectively. Subscript ref means the reference values.

Table 4. The results of iteration number (Ite Num.), and the utilities of the demander (De.) and supplier (Su.) for various combinations of $(\kappa, \beta)$

\begin{tabular}{llllllllll}
$\beta$ & 0.2 & 0.4 & 0.6 & 0.8 & 1.0 & 1.2 & 1.4 & 1.6 & 1.8 \\
$\kappa$ & 0.8 \\
\hline
\end{tabular}




\begin{tabular}{ccccccccccc}
\hline \multirow{4}{*}{0.1} & Ite Num. & 100 & 100 & 97 & 91 & 85 & 79 & 74 & 69 & 65 \\
& De. & 0.1295 & 0.2255 & 0.2217 & 0.2255 & 0.2247 & 0.2232 & 0.2209 & 0.2247 & 0.2211 \\
& Su. & 0.1249 & 0.2003 & 0.2881 & 0.2714 & 0.2740 & 0.2714 & 0.2809 & 0.2757 & 0.2843 \\
0.2 & Ite Num. & 100 & 99 & 93 & 85 & 78 & 72 & 66 & 61 & 57 \\
& De. & 0.1716 & 0.2188 & 0.2278 & 0.2247 & 0.2247 & 0.2255 & 0.2211 & 0.2247 & 0.2217 \\
& Su. & 0.1680 & 0.2687 & 0.2573 & 0.2757 & 0.2809 & 0.2687 & 0.2843 & 0.2809 & 0.2863 \\
& Ite Num. & 100 & 96 & 87 & 78 & 70 & 63 & 58 & 53 & 49 \\
0.3 & De. & 0.2015 & 0.2261 & 0.2209 & 0.2211 & 0.2211 & 0.2232 & 0.2217 & 0.2211 & 0.2211 \\
& Su. & 0.2003 & 0.2757 & 0.2809 & 0.2843 & 0.2843 & 0.2779 & 0.2881 & 0.2843 & 0.2843 \\
& Ite Num. & 100 & 91 & 79 & 68 & 60 & 54 & 48 & 44 & 40 \\
0.4 & De. & 0.2255 & 0.2255 & 0.2247 & 0.2225 & 0.2232 & 0.2247 & 0.2232 & 0.2211 & 0.2232 \\
& Su. & 0.2392 & 0.2726 & 0.2779 & 0.2809 & 0.2843 & 0.2779 & 0.2809 & 0.2863 & 0.2809 \\
& Ite Num. & 97 & 79 & 65 & 54 & 47 & 41 & 37 & 33 & 30 \\
0.5 & De. & 0.2255 & 0.2225 & 0.2217 & 0.2225 & 0.2217 & 0.2217 & 0.2247 & 0.2217 & 0.2211 \\
& Su. & 0.2687 & 0.2843 & 0.2881 & 0.2843 & 0.2881 & 0.2863 & 0.2809 & 0.2863 & 0.2843 \\
& Ite Num. & 79 & 54 & 41 & 32 & 27 & 23 & 21 & 19 & 17 \\
0.6 & De. & 0.2225 & 0.2225 & 0.2232 & 0.2225 & 0.2217 & 0.2225 & 0.2232 & 0.2247 & 0.2217 \\
& Su. & 0.2843 & 0.2843 & 0.2843 & 0.2843 & 0.2863 & 0.2843 & 0.2809 & 0.2809 & 0.2881 \\
\hline
\end{tabular}

The relative error of the utilities is illustrated in figure 8(a). To be clear, both three-dimensional and two-dimensional results are shown. For the combination of small $\kappa$ and $\beta$, relative error could be very large, up to 100 percents. With the increase of $\kappa$ and $\beta$, the error decrease gradually. Less than $5 \%$ error is observed when $\kappa$ and $\beta$ is large enough. Recall the results in table 4 , it seems that larger values of $\kappa$ and $\beta$ usually behave better. However, it should be remembered that very large $\kappa$ may lead to a crash of the program, as mentioned before.

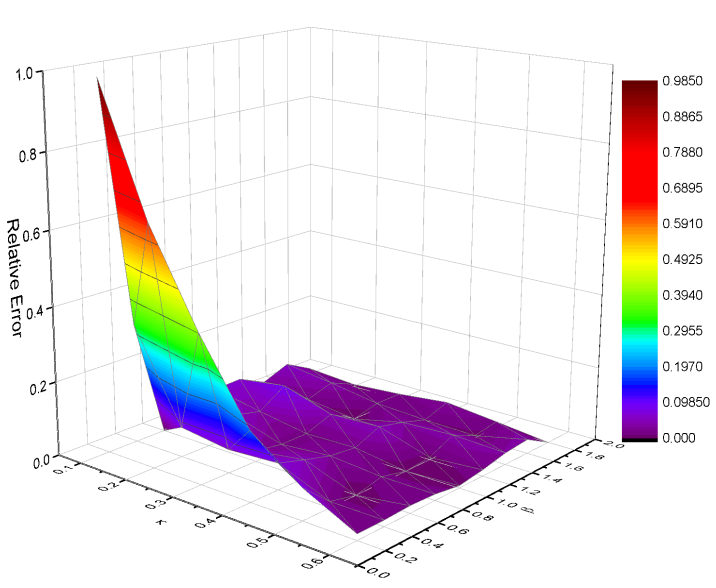

(a) Three-dimensional

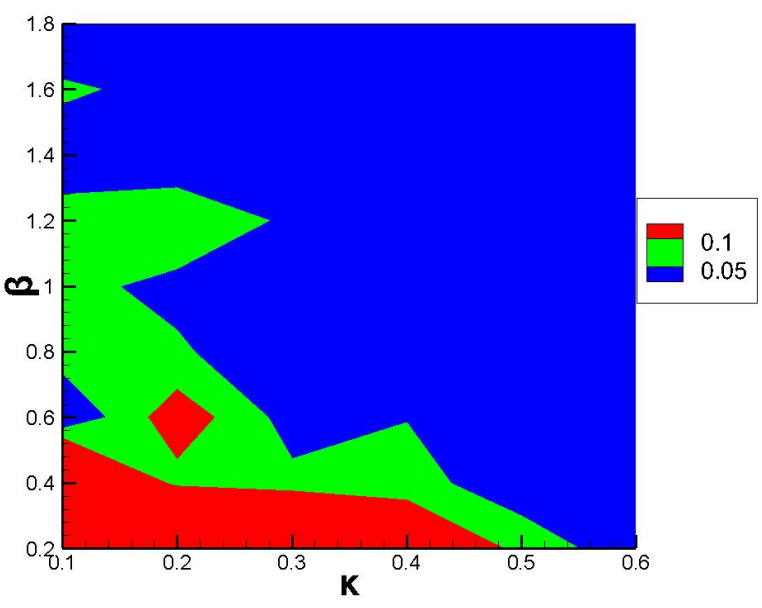

(b) Two-dimensional

Figure 8. Relative error of the utilities in (a) three-dimensional and (b) two-dimensional.

\section{Conclusions}

An innovation service pricing model that is based on a combination of the genetic algorithm and negotiation is proposed in this paper. Based on three hypotheses, the model is analyzed and interpreted in detail. The model utilizes the utility function of commonly considered parameters of the service supplier and demander as the objective function, identifies the Pareto boundary via NSGA-II, and adopts a learning strategy as the negotiation strategy. The model is utilized to study the pricing process of an R \& D service, and the optimal price is successfully identified. Therefore, the reliability of the model is demonstrated. 
A detailed discussion on the influence of the learning strategy and some user-defined parameters during the negotiation process are performed based on the selected implementation. It is observed that the proposed learning strategy greatly accelerate the negotiation process with nearly no influence on the final results. In addition, a a larger initial concession parameter $\kappa$ and concession speed control parameter $\beta$ will lead to a smaller iteration number with acceptable accuracy obtained. While the initial concession parameter $\kappa$ should not be too large, otherwise the program will crash.

However, the model has various shortcomings. For example, in this model, the negotiation process is only affected by the behavior of the negotiating counterparty, and the influence of the market supply-demand environment is not considered. This shortcoming will be resolved in the future so that the pricing model can better adapt to the development of innovation services.

Author Contributions: Conceptualization, Y.Z. and Y.L.; methodology, Y.Z. and Y.X.Z; software, Y.X.Z.; writing-original draft preparation, Y.Z.; writing-review and editing, Y.L. and Y.X.Z.; supervision, Y.L.; funding acquisition, Y.L.

Funding: This research was funded by the National Natural Science Foundation of China (No. 71704036), the Harbin University of Science and Technology's 2019 "Science and Engineering Talents" Program Outstanding Young Talent Project (2019-KYYWF-0216), and the Harbin University of Science and Technology School of Economics and Management "Double First Class" Discipline Construction High-level Cultivation Project (KY202013C).

Acknowledgments: The authors are grateful for the important and valuable comments of anonymous reviewers.

Conflicts of Interest: The authors declare no conflict of interest.

\section{References}

1. Sheng, M.K.; Luo, J. Comparative Research on Strategy and Policy of Science and Technology Innovation between China and India - Take STI in India and China's "Outline of Nation Innovation-Driven Develoment Strategy" as an Example. Science \& Technology progress and policy, 2018, 35, 127-134. [DOI: 10.6049/kjjbydc.2017110309]

2. Li, Y.; Guo, H.; Cooper, S.Y.; et al. The Influencing Factors of the Technology Standard Alliance Collaborative Innovation of Emerging Industry. Sustainability, 2019, 11, 1-17. [DOI: 10.3390/su11246930]

3. Wu, J.; Yang, Z.; Hu, X.; et al. Exploring Driving Forces of Sustainable Development of China's New Energy Vehicle Industry: An Analysis from the Perspective of an Innovation Ecosystem. Sustainability, 2018, 10, 1-24. [DOI: 10.3390/su10124827]

4. Villani, E.; Rasmussen, E.; Grimaldi, R.; et al. How Intermediary Organizations Facilitate University Industry Technology Transfer: A Proximity Approach. Technol. Forecast. Soc. Chang., 2017, 114, 86-102. [DOI: 10.1016/j.techfore.2016.06.004]

5. Stezano, F. The Role of Technology Centers as Intermediary Organizations Facilitating Links for Innovation: Four Cases of Federal Technology Centers in Mexico. Rev. Policy Res., 2018, 35, 642-666. [DOI: 10.1111/ropr.12293]

6. Fu, W.; Wang, Q.; Zhao, X.; et al. The Influence of Platform Service Innovation on Value Co-creation Activities and the Network Effect. J. Serv. Manage., 2017, 28, 348-388. [DOI: 10.1108/JOSM-10-2015-0347]

7. Hong, K.; Hu, J.R.; Wang, W. Research on the Innovative Clusters and Countermeasures of Scince \& Technology Information Resource Sharing Platform Kunming, Chengdu Platform as an Example. 5th International Conference on Cooperation and Promotion of Information Resources in Science and Technology, 2010, 386-391.

8. Abbate, T.; Codini, A.P.; Aquilani, B.; et al. Knowledge Co-creation in Open Innovation Digital Platforms: Processes, Tools and Services. J. Bus. Ind. Mark., 2019, 34, 1434-1447. [DOI: 10.1108/JBIM-09-2018-0276]

9. Gronroos, C. Value Co-creation in Service Logic: A Critical Analysis. Mark. Theory, 2011, 11, 279-301. [DOI: 10.1177/1470593111408177]

10. Cui, F.; Tang, H.; Zhang, Q.; et al. Integrating Ecosystem Services Supply and Demand into Optimized Management at Different Scales: A Case Study in Hulunbuir, China. Ecosyst. Serv., 2019, 39. [DOI: 10.1016/j.ecoser.2019.100984] 
11. Kung, L.; Zhong, G. The Optimal Pricing Strategy for Two-Sided Platform Delivery In the Sharing Economy. Transp. Res. Pt. e-Logist. Transp. Rev., 2017, 1-12. [DOI: 10.1016/j.tre.2017.02.003]

12. Li, H.; Dong, M.; Ota, K.; et al. Pricing and Repurchasing for Big Data Processing in Multi-Clouds. IEEE Trans. Emerg. Top. Comput., 2016, 4, 266-277. [DOI: 10.1109/TETC.2016.2517930]

13. Choi, T.; Guo, S.; Liu, N.; et al. Optimal Pricing in On-Demand-Service-Platform-Operations with Hired Agents and Risk-Sensitive Customers in the Blockchain Era. Eur. J. Oper. Res., 2020, 284, 1031-1042. [DOI: 10.1016/j.ejor.2020.01.049]

14. Kohtamaki, M.; Partanen, J. Co-creating Value from Knowledge-intensive Business Services in Manufacturing Firms: The Moderating Role of Relationship Learning in Supplier-customer Interactions. J. Bus. Res., 2016, 69, 2498-2506. [DOI: 10.1016/j.jbusres.2016.02.019]

15. Raiffa, H. The Art and Science of Negotiation. Harvard University Press, 1982.

16. De Bock, K.W.; Coussement, K.; Lessmann, S.; et al. Cost-sensitive Business Failure Prediction when Misclassification Costs are Uncertain: A Heterogeneous Ensemble Selection Approach. Eur. J. Oper. Res., 2020. [DOI: 10.1016/j.ejor.2020.01.052]

17. Babaei, G.; Bamdad, S. A Multi-objective Instance-based Decision Support System for Investment Recommendation in Peer-to-peer Lending. Expert Syst. Appl., 2020. [DOI: 10.1016/j.eswa.2020.113278]

18. Mavrovouniotis, M.; Li, C.; Yang, S.; et al. A Survey of Swarm Intelligence for Dynamic Optimization: Algorithms and Applications. Swarm Evol. Comput., 2017, 1-17. [DOI: 10.1016/j.swevo.2016.12.005]

19. Li, A.; Xue, B.; Zhang, M.; et al. Multi-objective Feature Selection Using Hybridization of a Genetic Algorithm and Direct Multisearch for Key Quality Characteristic Selection. Inf. Sci., 2020, 245-265. [DOI: 10.1016/j.ins.2020.03.032]

20. Shirvani, M.H. Bi-objective Web Service Composition Problem in Multi-cloud Environment: A Bi-objective Time-varying Particle Swarm Optimisation Algorithm. J. Exp. Theor. Artif. Intell., 2020, 1-24. [DOI: 10.1080/0952813X.2020.1725652]

21. Srinivas, N.; Deb, K. Multiobjective Function Optimization Using Nondominated Sorting Genetic Algorithms, Evolut. Comput, 1995, 2, 221-248. [DOI: 10.1162/evco.1994.2.3.221]

22. Deb, K.; Pratap, A.; Agarwal, S.; et al. A Fast and Elitist Multiobjective Genetic Algorithm: NSGA-II. IEEE Trans. Evol. Comput., 2002, 6, 182-197. [DOI: 10.1109/4235.996017]

23. Faratin, P.; Sierra, C.; Jennings, N. R. Negotiation Decision Functions for Autonomous Agents. Robot. Auton. Syst., 1998, 24, 159-182. [DOI: 10.1016/S0921-8890(98)00029-3]

24. Hindriks, K.V.; Tykhonov, D.; De Weerdt, M.; et al. Qualitative One-to-Many Multi-Issue Negotiation: Approximating the QVA. Group Decis. Negot., 2012, 21, 49-77. [DOI: 10.1016/S0921-8890(98)00029-3] 